\title{
Angles-only Navigation to a Non-Cooperative Satellite using Relative Orbital Elements
}

\author{
G. Gaias*, S. D’Amico', and J.-S. Ardaens* \\ German Aerospace Center (DLR), Münchner Str. 20, 82234 Wessling, Germany
}

\begin{abstract}
This work addresses the design and implementation of a prototype relative navigation tool that uses camera-based measurements collected by a servicer spacecraft to perform farrange rendezvous with a non-cooperative client in low Earth orbit. The development serves the needs of future on-orbit-servicing missions planned by the German Aerospace Center. The focus of the paper is on the design of the navigation algorithms and the assessment of the expected performance and robustness under real-world operational scenarios. The tool validation is accomplished through a high-fidelity simulation environment based on the Multi-Satellite-Simulator in combination with the experience gained from actual flight data from the GPS and camera systems on-board the PRISMA mission.
\end{abstract}

\section{Introduction}

This work addresses the design and implementation of a prototype relative navigation tool that makes use of camera-based measurements only. Its development was mainly motivated by the need to build knowhow for vision-based navigation and prepare for future on-orbit servicing missions of the German Aerospace Center (DLR) such as DEOS (DEutsche Orbitale Servicing). ${ }^{1}$ In this class of space missions, a servicer satellite has to approach a client non-cooperative spacecraft from far range distance. The capability to safely accomplish this with no need of a device that directly measures the range distance represents an asset for the servicer's system design. Although several theoretical studies have been conducted on anglesonly relative navigation, a solid assessment of the expected performance and robustness under real-world rendezvous scenarios, being them with ground-in-the-loop or fully autonomous, is needed. This paper takes advantage of the DLR's contributions to the Swedish PRISMA mission ${ }^{2,3}$ and makes use of actual flight data collected during the related technology demonstrations to validate a novel non-cooperative relative navigation prototype. Among the several sensors on-board, the servicer spacecraft of PRISMA is also equipped with a star tracker developed by the Danish Technical University (DTU) called Vision Based System (VBS) which is able to provide the line-of-sight (LOS) measurement to a target non-stellar object. User experience, image data, and GPS precise orbit determination results acquired during the DLR's experiments are a most valuable resource for the design of the navigation approach and for the prediction of related performances.

The first part of the work deals with the filter design. Here the problem is described in terms of available measurements and aimed state to be reconstructed. Measurements consist of azimuth and elevation of the LOS to the client satellite. The relative dynamics is described through relative orbital elements. Such a choice is justified by the clear geometrical insight that these elements provide to understand the relative geometry and, therefore, the LOS behavior. Moreover, relative orbital elements allow the straightforward inclusion of Earth oblateness effects due to the $J_{2}$ in the relative motion model. ${ }^{4}$ Finally the un-observability of the relative navigation problem is mostly condensed in only one component of the state: the relative mean argument of latitude. Therefore the shape of the relative motion can be determined from the very early phases of the approach based on the observable relative orbital elements. The estimation error of the relative mean argument of latitude is stepwise decreased while executing a maneuvers' profile. Nevertheless the knowledge of the geometry allows performing safe approaches simply by setting a proper relative eccentricity and inclination vector separation.

\footnotetext{
*Research Engineer, GSOC/Space Flight Technology, Münchner Str. 20, 82234 Wessling, Germany.

${ }^{\dagger}$ Lead Research Engineer, GSOC/Space Flight Technology, Münchner Str. 20, 82234 Wessling, Germany.
} 
As well-known from the literature, the angles-only navigation problem is not fully observable in the absence of maneuvers. Sufficient conditions to achieve observability are provided in Ref. 6,7 for the bearingonly target motion analysis. In the field of orbital rendezvous, they are presented in Ref. 8. According to these contributions, a maneuver that produces a variation of the LOS natural trend makes the system observable. In this work it is shown that relative orbital elements can support a direct physical interpretation of the un-observability issues. Moreover results are generalized for the case in which the perturbations due to the equatorial bulge are taken into account. It is here shown that observability properties improve for certain families of relative orbits as the trends of the mean relative elements deviate from the one they have within the Kepler assumptions. The exploitation of relative orbital elements is of great benefit also when dealing with the assessment of the estimation accuracy achievable when maneuvers are performed. In the literature different methods to quantify the observability of the problems are suggested. They span from the definition of a range error metric, ${ }^{9}$ to the exploitation of the covariance of the estimated state ${ }^{10}$ or of the condition number of the observability matrix. ${ }^{11}$ In our work simple metrics are proposed to quantify the effectiveness of the maneuvers performed. They are based on the immediate idea of what changes will take place in the LOS behavior due to the relative orbital elements variation determined by a maneuver. In this frame an optimization problem can be easily set up.

The validation of the tool has been accomplished through the Multi-Satellite Simulator employed at DLR/GSOC to support various projects in the fields of formation flying and proximity operations. ${ }^{12}$ Specifically, a high-fidelity simulation environment provides all the information usually available in the telemetry of the servicer satellite. A camera model mimics the measurement data delivered by an image processing unit. Its performances are tailored according to the processing of actual PRISMA VBS image data, collected during the DLR's Formation Reacquisition experiment(August 2011). ${ }^{13}$ Modeled measurement data, servicer absolute information together with the estimated maneuver plan are used by the navigation tool to perform relative orbit determination. As a result, the simulations provide the typical navigation accuracy which is achievable through a specific maneuver profile under realistic operational conditions. The proposed navigation tool contributes to the preparation to the DLR/GSOC Advanced Rendezvous Demonstration using GPS and Optical Navigation (ARGON) experiment, scheduled for the extended phase of the PRISMA mission. ${ }^{14}$

\section{Mathematical Description}

The relative orbit determination problem consists in estimating at a certain time the relative state of a client satellite with respect to the servicer one, making use of a set of observations, given that the initial relative state is unknown and an a-priori first guess might be available. In the case of angles-only measurements, observations at each instant of time consist of a couple of angles, i.e. azimuth $\eta$ and elevation $\psi$, which subtend the line-of-sight LOS unit-vector, $u^{\mathrm{c}}$, to the client satellite. The superscript "c" denotes the camera frame, i.e. the frame attached to the sensor, and angles are modeled according to the following nonlinear functions of the relative state $\mathbf{x}$ :

$$
\left(\begin{array}{c}
\eta \\
\psi
\end{array}\right)=\left(\begin{array}{c}
\arctan \left(u_{x}^{\mathrm{c}} / u_{z}^{\mathrm{c}}\right) \\
\arcsin \left(u_{y}^{\mathrm{c}}\right)
\end{array}\right)=\mathbf{z}(t, \mathbf{x}(t))
$$

The nonlinear differential equations governing the relative dynamics simplifies to linear differential equations under the assumptions of relative separation much smaller than the servicer absolute radius and servicer satellite on a circular orbit.

$$
\dot{\mathbf{x}}(t)=\hat{\mathbf{A}}(t) \mathbf{x}(t)+\hat{\mathbf{B}}(t) d \mathbf{v}(t)
$$

The solution of the obtained set of linear differential equations can be expressed via the state transition matrix that maps the relative state vector from one time to another. The nonlinear measurements equations Eq.(1) can be linearized about a nominal reference relative state $\mathbf{x}^{r e f}$. This procedure leads to the following set of functions in the presence of control inputs and measurements uncertainties:

$$
\begin{aligned}
& \mathbf{x}(t)=\hat{\boldsymbol{\Phi}}\left(t, t_{0}\right) \mathbf{x}\left(t_{0}\right)+\int_{t_{0}}^{t} \hat{\boldsymbol{\Phi}}(t, \tau) \hat{\mathbf{B}}(\tau) d \mathbf{v}(\tau) d \tau \\
& \mathbf{y}(t)=\hat{\mathbf{C}}(t) \mathbf{x}(t)+\boldsymbol{\epsilon}(t)
\end{aligned}
$$


Concerning the relative orbit estimation problem, the complete estimation state is composed by:

$$
\mathbf{x}=\left(\begin{array}{c}
a^{\mathrm{o}} \delta \boldsymbol{\alpha} \\
\mathbf{b}
\end{array}\right)
$$

Where the relative state is parameterized in terms of relative orbital elements according to:

$$
\delta \boldsymbol{\alpha}=\left(\begin{array}{c}
\delta a \\
\delta e_{x} \\
\delta e_{y} \\
\delta i_{x} \\
\delta i_{y} \\
\delta u
\end{array}\right)=\left(\begin{array}{c}
\delta a \\
\delta e \cos \varphi \\
\delta e \sin \varphi \\
\delta i \cos \theta \\
\delta i \sin \theta \\
\delta u
\end{array}\right)=\left(\begin{array}{c}
\left(a-a^{\mathrm{o}}\right) / a^{\mathrm{o}} \\
e \cos \omega-e^{\mathrm{o}} \cos \omega^{\mathrm{o}} \\
e \sin \omega-e^{\mathrm{o}} \sin \omega^{\mathrm{o}} \\
i-i^{\mathrm{o}} \\
\left(\Omega-\Omega^{\mathrm{o}}\right) \sin i^{\mathrm{o}} \\
u-u^{\mathrm{o}}
\end{array}\right)
$$

Here $a, e, i, \omega, \Omega$, and $M$ denote the classical Keplerian elements, whereas $\mathbf{e}=(e \cos \omega e \sin \omega)^{\mathrm{T}}$, and $u=M+\omega$ represent the eccentricity vector and the mean argument of latitude. Here the superscript "o" denotes quantities referring to the reference spacecraft which defines the origin of the orbital frame (here the servicer). From now on, the absolute orbital elements which appear in the equations will always belong to the servicer satellite, therefore the superscript is dropped. Under the assumptions of the Hill-Clohessy-Wilshire equations $(\mathrm{HCW}),{ }^{15}$ the magnitude of the relative eccentricity and inclination vectors, $\delta \mathbf{e}$ and $\delta \mathbf{i}$, provide the amplitudes of the in-plane and out-of-plane relative motion oscillations, whereas relative semi-major axis, $\delta a$, and relative mean longitude, $\delta \lambda=\delta u+\delta i_{y} \cot i$, provide mean offsets in radial and along-track directions respectively. ${ }^{5,4}$ The orientation of the shape of the relative motion is driven by the phase angles $\varphi$ and $\theta$, which identify the relative perigee and relative ascending node of the relative orbit.

The last two components of the estimation state are represented by the biases of the sensor:

$$
\mathbf{b}=\left(\begin{array}{c}
b_{\eta} \\
b_{\psi}
\end{array}\right)
$$

Given Eqs.(4),(6), the matrix quantities that compare in Eqs.(2),(3) are composed by the following subparts:

$$
\left.\begin{array}{ll}
\hat{\mathbf{A}}(t)=\left(\begin{array}{ll}
\mathbf{A}(t) & \mathbf{O}_{6 \times 2} \\
\mathbf{O}_{2 \times 6} & \mathbf{O}_{2 \times 2}
\end{array}\right) \quad, \quad \hat{\mathbf{\Phi}}\left(t, t_{0}\right)=\left(\begin{array}{cc}
\mathbf{\Phi}\left(t, t_{0}\right) & \mathbf{O}_{6 \times 2} \\
\mathbf{O}_{2 \times 6} & \mathbf{\Phi}_{b}\left(t, t_{0}\right)
\end{array}\right) \\
\hat{\mathbf{B}}(t) \\
\mathbf{O}_{2 \times 3}
\end{array}\right) \quad, \quad \hat{\mathbf{C}}(t)=\left(\begin{array}{cc}
\mathbf{C}(t) & \mathbf{I}_{2 \times 2}
\end{array}\right)
$$

Therefore, the $8 \times 8$ state transition matrix $\hat{\mathbf{\Phi}}$ relates the 8 -dimensional state vector $\mathbf{x}\left(t_{0}\right)$ at time $t_{0}$ to the state $\mathbf{x}(t)$ at time $t$. The $6 \times 3$ control input matrix $\mathbf{B}$ expresses the variation of the relative orbital elements caused by an impulsive maneuver $\Delta \mathbf{v}=\left(\Delta v_{\mathrm{R}} \Delta v_{\mathrm{T}} \Delta v_{\mathrm{N}}\right)^{\mathrm{T}}$ at time $t_{\mathrm{M}}$ with components in radial, along-track, and cross-track directions. The partial derivatives of the modeled angle measurements $\mathbf{z}$ with respect to the state are given by the $2 \times 8$ measurement sensitivity matrix $\hat{\mathbf{C}}$. $\boldsymbol{\epsilon}$ is the two-dimensional vector of uncorrelated measurement errors characterized by a normal distribution with zero mean and covariance $\operatorname{Cov}(\boldsymbol{\epsilon})=\mathbf{W}=\operatorname{diag}\left(\sigma_{\eta}^{2}, \sigma_{\psi}^{2}\right)$.

Regarding the system of equations Eq.(2) that lead to Eq.(3), a simple relative dynamics model which captures the most relevant perturbations in low Earth orbit is employed. ${ }^{5,4}$ Under the assumptions of Kepler orbits the relative state of Eq.(5) is composed by the integration constants of the HCW equations. Therefore, for orbits of equal energy (i.e., $\delta a=0), \boldsymbol{\Phi}\left(t, t_{0}\right)=\mathbf{I}_{6 \times 6}$. When the servicer and client satellites have unequal semi-major axis (e.g., $\delta a>0$ ), the relative mean argument of latitude changes over time. If $\delta a$ and $\delta u$ are small quantities (i.e., $\delta a$ and $\delta u<<1$ ), its drift can be expressed to first order as $\mathbf{A}_{6,1}=-1.5 n$, being $n$ the mean motion of the servicer. Thus the particular choice of the variable state allows having the following simple relationship between the matrix of the dynamics of the system $\mathbf{A}(t)$ and the state transition matrix $\mathbf{\Phi}\left(t, t_{0}\right)$ :

$$
\mathbf{\Phi}\left(t, t_{0}\right)=\mathbf{I}_{6 \times 6}+\left(t-t_{0}\right) \cdot \mathbf{A}(t)
$$


Earth oblateness effects due to $J_{2}$ can be easily introduced in the relative dynamics model under the additional assumptions of small magnitudes of the relative eccentricity/inclination vectors (i.e., $\delta e$ and $\delta i<<1$ ) and small eccentricity (i.e., $e<<1$ ). The resulting secular variations of the relative orbital elements can be derived from the theory of Brower. ${ }^{16}$ These effects are proportional to the elapsed time $\Delta t$ and $J_{2}$ and are expressed through to the following parameters:

$$
\begin{aligned}
& \dot{\varphi}=1.5 \gamma n\left(5 \cos ^{2} i-1\right) \\
& \gamma=0.5 J_{2}\left(\mathrm{R}_{\mathrm{E}} / a\right)^{2}
\end{aligned}
$$

where $R_{E}$ is the Earth equatorial radius. Specifically, the Earth equatorial bulge causes a rotation of the relative eccentricity vector, $\delta$ e (i.e. $\mathbf{A}_{2,3}$ and $\mathbf{A}_{3,2}$ ), a vertical linear drift of the relative inclination vector, $\delta \mathbf{i}$ (i.e. $\mathbf{A}_{5,4}$ ), and a linear drift of $\delta u$ (i.e. $\mathbf{A}_{6,4}$ ). The inclusion of the aforementioned effects leads to the following complete state transition matrix:

$$
\boldsymbol{\Phi}\left(t, t_{0}\right)=\left(\begin{array}{cccccc}
1 & 0 & 0 & 0 & 0 & 0 \\
0 & 1 & -\dot{\varphi} \Delta t & 0 & 0 & 0 \\
0 & \dot{\varphi} \Delta t & 1 & 0 & 0 & 0 \\
0 & 0 & 0 & 1 & 0 & 0 \\
0 & 0 & 0 & 3 \gamma \sin ^{2}(i) n \Delta t & 1 & 0 \\
-1.5 n \Delta t & 0 & 0 & -12 \gamma \sin (2 i) n \Delta t & 0 & 1
\end{array}\right)
$$

Biases are modeled as esponentially correlated measurement errors, therefore their time evolution is expressed by the following state transition matrix:

$$
\boldsymbol{\Phi}_{b}\left(t, t_{0}\right)=\left(\begin{array}{cc}
\exp (-|\Delta t| / \tau) & 0 \\
0 & \exp (-|\Delta t| / \tau)
\end{array}\right)
$$

where $\tau$ is the correlation time constant. Whenever $\tau=\infty$, then the modeling of the biases simplifies to un-correlated measurement errors.

The variation of the relative orbital elements caused by an impulsive maneuver (or an instantaneous velocity change) at time $t_{\mathrm{M}}$ can be modeled under the same assumptions of our linear relative dynamics. In particular the inversion of the solution of the HCW equations expressed in terms of relative orbital elements provides the following relationship: ${ }^{5,4}$

$$
\mathbf{B}\left(t_{\mathrm{M}}\right)=-\frac{1}{n}\left(\begin{array}{ccc}
0 & 2 & 0 \\
\sin u_{\mathrm{M}} & 2 \cos u_{\mathrm{M}} & 0 \\
-\cos u_{\mathrm{M}} & 2 \sin u_{\mathrm{M}} & 0 \\
0 & 0 & \cos u_{\mathrm{M}} \\
0 & 0 & \sin u_{\mathrm{M}} \\
-2 & 0 & -\sin u_{\mathrm{M}} \cot i
\end{array}\right)
$$

where $u_{\mathrm{M}}=u\left(t_{\mathrm{M}}\right)$ identifies the mean argument of latitude of the servicer at the delta-v time. Eq.(12) shows how along-track maneuvers cause instantaneous variations of $\delta a$ and $\delta \mathbf{e}$, radial maneuvers cause instantaneous variations of $\delta u$ and $\delta \mathbf{e}$, whereas cross-track maneuvers affect $\delta u$ and $\delta \mathbf{i}$. It is noted that crosstrack maneuvers do not change the mean along-track separation, $\delta \lambda=\delta u+\delta i_{y} \cot i$, because the effects on $\delta u$ and $\delta i_{y}$ cancel out, thus the in-plane and out-of-plane relative motion remain fully decoupled in this formulation. Given Eqs.(10),(12), the zero state transition of the system, i.e. the integral in Eq.(3), can be immediately solved.

The partial derivatives of the angle measurements w.r.t. relative orbital elements about the reference state are computed through the application of the following chain rule:

$$
\begin{aligned}
\mathbf{C}(t)=\left.\frac{\partial \mathbf{z}}{\partial \delta \boldsymbol{\alpha}}\right|_{\overline{\delta \boldsymbol{\alpha}}} & =\left.\frac{\partial \mathbf{z}}{\partial \delta \mathbf{r}^{\mathrm{c}}} \cdot \frac{\partial \delta \mathbf{r}^{\mathrm{c}}}{\partial \delta \mathbf{r}^{\mathrm{J} 2000}} \cdot \frac{\partial \delta \mathbf{r}^{\mathrm{J} 2000}}{\partial \delta \mathbf{r}^{\mathrm{RTN}}} \cdot \frac{\partial \delta \mathbf{r}^{\mathrm{RTN}}}{\partial \delta \boldsymbol{\alpha}}\right|_{\overline{\delta \boldsymbol{\alpha}}} \\
& =\left.\left(\frac{\partial \eta}{\partial \delta \mathbf{r}^{\mathrm{c}}} \frac{\partial \psi}{\partial \delta \mathbf{r}^{\mathrm{c}}}\right)^{\mathrm{T}} \cdot \mathbf{R}_{\mathrm{J} 2000}^{\mathrm{c}} \cdot \mathbf{R}_{\mathrm{RTN}}^{\mathrm{J} 2000} \cdot \frac{\partial \delta \mathbf{r}^{\mathrm{RTN}}}{\partial \delta \boldsymbol{\alpha}}\right|_{\overline{\delta \boldsymbol{\alpha}}}
\end{aligned}
$$


Here usage has been made of the fact that the derivative of the angle measurements w.r.t. the relative velocity $\partial \mathbf{z} / \partial \delta \mathbf{v}^{\mathrm{c}}$ is zero. The expansion given by Eq.(13) contains the following main terms, namely 1) the derivative of the azimuth and elevation angles w.r.t. relative position in the camera frame $\left.\delta \mathbf{r}^{\mathrm{c}}, 2\right)$ the absolute attitude of the sensor, that is the rotation matrix from J2000 to the camera frame, 3) the rotation matrix from the RTN frame to the inertial frame, and 4) the derivatives of the relative position in the RTN frame $\delta \mathbf{r}^{\text {RTN }}$ w.r.t. the relative orbital elements $\delta \boldsymbol{\alpha}$. The mapping between relative orbital elements and relative position in the orbital frame is given by the adopted linear model as: $:^{5,4}$

$$
\left.\frac{\partial \delta \mathbf{r}^{\mathrm{RTN}}}{\partial \delta \boldsymbol{\alpha}}\right|_{\overline{\delta \boldsymbol{\alpha}}}=\left(\begin{array}{cccccc}
1 & -\cos u & -\sin u & 0 & 0 & 0 \\
0 & 2 \sin u & -2 \cos u & 0 & \cot i & 1 \\
0 & 0 & 0 & \sin u & -\cos u & 0
\end{array}\right)
$$

The measurements partials w.r.t. relative position in the camera frame can be computed using the following equivalence: ${ }^{17}$

$$
\frac{\partial \delta \mathbf{r}^{c}}{\partial \delta \mathbf{r}^{c}}=\mathbf{I}_{3 x 3}=\mathbf{u}^{\mathrm{c}} \frac{\partial \delta r}{\partial \delta \mathbf{r}^{c}}+\delta r \frac{\partial \mathbf{u}^{\mathrm{c}}}{\partial \eta} \frac{\partial \eta}{\partial \delta \mathbf{r}^{c}}+\delta r \frac{\partial \mathbf{u}^{\mathrm{c}}}{\partial \psi} \frac{\partial \psi}{\partial \delta \mathbf{r}^{c}}
$$

where appear the three orthogonal directions: LOS unit-vector, $\left(\partial \mathbf{u}^{\mathrm{c}} / \partial \eta\right)$ and $\left(\partial \mathbf{u}^{\mathrm{c}} / \partial \psi\right)$. Among these, the last two vectors are computed from Eq.(1):

$$
\begin{aligned}
& \frac{\partial \mathbf{u}^{\mathrm{c}}}{\partial \eta}=\left(\begin{array}{c}
\cos \psi \cos \eta \\
0 \\
-\cos \psi \sin \eta
\end{array}\right) \\
& \frac{\partial \mathbf{u}^{\mathrm{c}}}{\partial \psi}=\left(\begin{array}{c}
-\sin \psi \sin \eta \\
\cos \psi \\
-\sin \psi \cos \eta
\end{array}\right)
\end{aligned}
$$

Now the derivative of the azimuth and elevation angles w.r.t. relative position in the camera frame can be computed by alternatively pre-multiplying Eq.(15) by $\left(\partial \mathbf{u}^{\mathrm{c}} / \partial \eta\right)^{\mathrm{T}}$ and $\left(\partial \mathbf{u}^{\mathrm{c}} / \partial \psi\right)^{\mathrm{T}}$, as the contributions in the remnant orthogonal directions vanish. As a result one obtains:

$$
\begin{aligned}
\left.\frac{\partial \eta}{\partial \delta \mathbf{r}^{\mathrm{c}}}\right|_{\overline{\delta \boldsymbol{\alpha}}} & =\frac{1}{\delta r \cos ^{2}(\psi)}\left(\frac{\partial \mathbf{u}^{\mathrm{c}}}{\partial \eta}\right)^{\mathrm{T}} \\
\left.\frac{\partial \psi}{\partial \delta \mathbf{r}^{\mathrm{c}}}\right|_{\overline{\delta \boldsymbol{\alpha}}} & =\frac{1}{\delta r}\left(\frac{\partial \mathbf{u}^{\mathrm{c}}}{\partial \psi}\right)^{\mathrm{T}}
\end{aligned}
$$

At this stage all the quantities of Eq.(13) are known in order to compute $\mathbf{C}(t)$. Finally, the last part of the sensitivity matrix $\hat{\mathbf{C}}(t)$ is constituted by the identity sub-matrix that represents the partial derivatives of the angle measurements w.r.t. the estimated biases.

\section{A. Observability of the estimation problem based on angles-only measures}

The problem of estimating a relative motion between two satellites making use of angles-only measurements is not fully observable. Physically this phenomenon is produced by the fact that infinite relative orbits generate the same LOS unit-vector trend over time. Equivalently the lack of measurements on the range cannot allow univocally solving for the separation between the satellites. Some authors emphasize that with a proper choice of parameters the main characteristics of the geometry of the natural motion can be reconstructed despite this not complete observability. ${ }^{8,18}$ That means that shape and orientation of the orbits are identified, leaving the ambiguity on a scale factor, i.e. the size of the relative motion, that is function of the separation between the satellites. To this aspect, formulating the relative dynamics through the relative orbital elements of Eq.(5) is particularly convenient. In this way in fact the range ambiguity is almost condensed in only one term of the state, i.e. the relative mean argument of latitude. The magnitude of the average separation over an orbital period is given by:

$$
\left\|\int_{0}^{2 \pi} \delta \mathbf{r}^{\mathrm{c}}(u) d u\right\|=a \sqrt{\delta a^{2}+\delta \lambda^{2}}
$$


Therefore for bounded orbits $(\delta a=0)$ with $\delta i_{y}=0$, the average separation coincides with $a \delta u$. For $\delta u>>\delta a$, the mean argument of latitude well approximates the average range magnitude. In addition to this property and differently from other geometry-based parameterizations, relative orbital elements support a powerful though simple model of the dynamics, able to capture some of the most relevant perturbations in low Earth orbit. This topic was discussed in the previous section. Figure 1 provides a sketch of the meaning of the relative orbital elements. The relative motion of the client vehicle is mapped in the orbital frame centered on the servicer and aligned with the radial ( $\mathrm{R}$, positive in Zenith direction), along-track ( $\mathrm{T}$, positive in flight direction), and cross-track (N, normal to the orbital plane) directions. The along-track drift due to the different orbit energies is neglected for visualization purposes. In the bottom-left subplot it is presented a possible attitude configuration of the sensing instrument. According to the sketch, the client satellite is preceding the servicer along the flight direction and the local camera frame is aligned to the orbital frame in such a way to point its boresight $z^{\mathrm{c}}$ in anti-flight direction:

$$
\mathbf{R}_{\mathrm{J} 2000}^{\mathrm{c}}=\left(\begin{array}{ccc}
1 & 0 & 0 \\
0 & 0 & 1 \\
0 & -1 & 0
\end{array}\right) \cdot \mathbf{R}_{\mathrm{J} 2000}^{\mathrm{RTN}}=\boldsymbol{\Gamma} \cdot \mathbf{R}_{\mathrm{J} 2000}^{\mathrm{RTN}}
$$

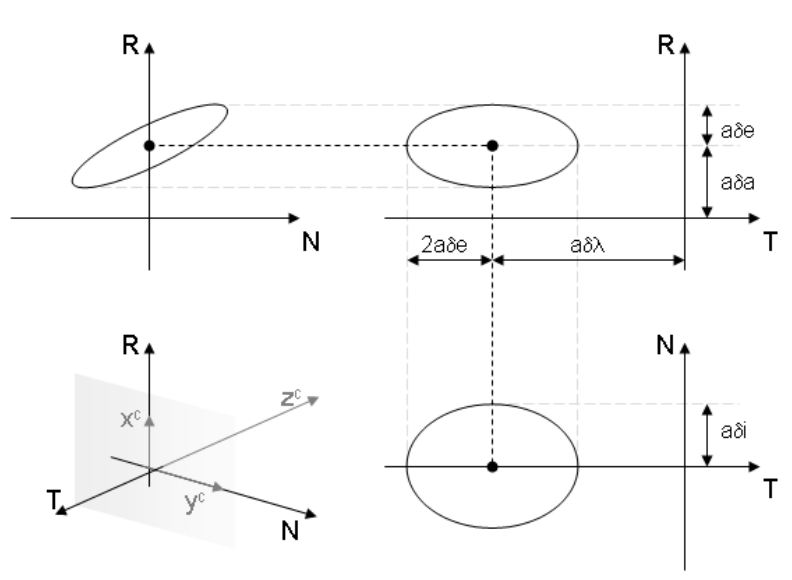

Figure 1: Description of the client relative motion in the servicer-centered orbital frame through relative orbital elements. The snapshot does not include the drift corresponding to $\delta a$. The bottom-left view presents a possible attitude of the sensor.

abservability techniques or local methods for the linearized problem have been investigated. In the sequel we focus only on results achieved within this second methodology.

In the field of bearing-only target motion analysis, Nardone and Aidala ${ }^{6}$ and Hammel and Aidala respectively developed the $2 \mathrm{D}$ and $3 \mathrm{D}$ necessary and sufficient conditions to ensure observability. In their working frame, a ship/submarine has to estimate the motion of a target which moves at constant absolute velocity. The relative dynamics is expressed through a linear model in the Cartesian relative position and velocity defined with respect to the active own-ship element. Nonlinear measurements equations, of a similar form of Eq.(1), are recast as linear expression in the unknown-initial state vector. Therefore a criterion for observability for linear time varying system is used. ${ }^{19}$ It exploits successive time derivatives of the reshaped measurements equation, in order to obtain a set of independent equations in the unknown-initial state vector.

In the field of orbital rendezvous, Woffinden and Geller ${ }^{8}$ developed an analytical form to ensure the sufficient conditions for the observability of the relative orbit determination problem. To accomplish this, they rewrite the nonlinear measurements equations in the form of LOS unit-vector function of the initialunknown Cartesian relative position and velocity. To this aim the state transition matrix of the linearized relative orbital motion is exploited. Subsequently observability criteria are deduced through geometrical 
considerations on the comparison of different measurement profiles generated starting from same initial conditions.

Both the criteria conclude that at least a maneuver is necessary in order to achieve the complete observability. Moreover such a maneuver has to produce a change in the relative position not aligned with the instantaneous direction of the natural LOS profile. In developing these criteria the formulation of the relative motion via Cartesian coordinates was exploited. Angles measurements and LOS unit-vector, in fact, can be expressed as not too complicated functions of the initial state vector. According to our formulation, the LOS unit-vector is given by:

$$
\begin{aligned}
\delta \mathbf{r}^{\mathrm{c}} & =\boldsymbol{\Gamma} \cdot \frac{\partial \delta \mathbf{r}^{\mathrm{RTN}}}{\partial \delta \boldsymbol{\alpha}} \cdot a \delta \boldsymbol{\alpha} \\
\mathbf{u}^{\mathrm{c}} & =\frac{\delta \mathbf{r}^{\mathrm{c}}}{\left\|\delta \mathbf{r}^{\mathrm{c}}\right\|}
\end{aligned}
$$

where the mapping matrix between relative orbital elements and relative position is Eq.(14) and the camera frame is oriented according to Eq.(19).

All the results achieved for the Cartesian relative state are valid for the state of Eq.(5) as long as the parameterization via relative orbital elements can be seen as the following change of state variable

$$
\left(\begin{array}{c}
\delta \mathbf{r}^{\mathrm{RTN}} \\
\delta \mathbf{v}^{\mathrm{RTN}}
\end{array}\right)=\left(\begin{array}{c}
\frac{\partial \delta \mathbf{r}^{\mathrm{RTN}}}{\partial \delta \boldsymbol{\alpha}} \\
\frac{\partial \delta \mathbf{v}^{\mathrm{RTN}}}{\partial \delta \boldsymbol{\alpha}}
\end{array}\right) \cdot a \delta \boldsymbol{\alpha}
$$

and both zero input response and zero state response are invariant to state transformations. In addition to this and as mentioned before, the utilization of relative orbital elements provides a direct physical interpretation that separates the observable portion of the state variable from the un-observable part. Numerically this can be proven by analyzing the behavior of the matrix of the accumulated partials of the measurements w.r.t. the initial state defined as follows:

$$
\mathbf{H}=\left(\begin{array}{c}
\mathbf{C}\left(t_{0}\right) \boldsymbol{\Phi}\left(t_{0}, t_{0}\right) \\
\vdots \\
\mathbf{C}\left(t_{n}\right) \boldsymbol{\Phi}\left(t_{n}, t_{0}\right)
\end{array}\right)
$$

In particular, if $\mathbf{H}$ has rank equal to 6 , the matrix $\mathbf{H}^{\mathrm{T}} \mathbf{H}$ is positive defined, thus invertible. Therefore the complete initial state can be reconstructed via the measurements that contributed in building $\mathbf{H}$. Let us first consider the same case discussed in the literature, i.e. the relative dynamics is represented by the HCW equations. Then, the transformed state transition matrix through Eq.(21) simplifies to the identity matrix with $\mathbf{I}_{6 \times 1}=1.5 n \Delta t$.

In Table 1 is introduced a set of relative orbits (RO) that represents a sample of typical relative motions; a brief description of their main characteristics is provided. They span from separations of $30 \mathrm{~km}$ to $100 \mathrm{~m}$. In particular RO1 represents a possible configuration for the beginning of an approach to a non-cooperative client satellite. RO2 presents a drift of almost $1 \mathrm{~km}$ towards the client, while keeping a safe separation in the $R-N$ plane. $^{20}$ RO3 can represent the hold-point were transition between far-range and close-range sensors can be performed. Finally RO4 can represent the starting point of a docking phase. In Table 2 are

Table 1: Set of typical relative orbits $(\mathrm{RO})$.

\begin{tabular}{lll} 
RO & Elements set $a \delta \boldsymbol{\alpha}[\mathrm{m}]$ & Description \\
\hline 1 & $(0,400,0,-400,0,-30000)$ & Bounded (anti)-parallel horizontal $\delta \mathbf{e} / \delta \mathbf{i}$ vectors \\
2 & $(-100,300,0,-300,0,-20000)$ & Drifting of circa $1 \mathrm{~km}$ per orbit towards the client \\
3 & $(0,0,-200,0,200,-3000)$ & Bounded (anti)-parallel vertical $\delta \mathbf{e} / \delta \mathbf{i}$ vectors \\
4 & $(0,0,0,0,0,-100)$ & V-bar station keeping
\end{tabular}

collected the rank and condition numbers achieved by these RO, when 6 measurements separated by the time correspondent to $30 \mathrm{deg}$ of variation of the servicer mean argument of latitude $\Delta u$ are accumulated. 
The size of $\mathbf{H}$ is $12 \times 6$ and the condition number of $\mathbf{H}^{\mathrm{T}} \mathbf{H}$ is used as a practical measurement criterion of the observability of the estimation problem, in agreement with Ref. 11 . There the value of $10^{16}$ is suggested as the limit of observability.

In Table 2, for each i-th RO, four cases are considered. In the first one, i.e. first row of each subblock, the state vector is represented by $\delta \boldsymbol{\alpha}$. In the other cases different sub-sets of the state variable are addressed: with "-" are marked the excluded relative elements. As expected, within the natural motion assumption, no RO achieves full rank. When the dimension of the state variable is reduced to 5, the observability properties variate substantially depending on which component is excluded. Specifically the best performance is obtained when the relative mean argument of latitude $\delta u$ is not estimated, see RO2. When the relative orbit to be estimated has no drift, i.e. $\delta a=0$, then $5 \mathrm{D}$ subsets that try to estimate $\delta u$ are not fully observable (rank equal to 4, i.e. RO 1, 3 and 4). In this case, in fact, infinite orbits produce the same LOS trend and the relative separation cannot be determined. Finally, the reconstruction of just the relative eccentricity and inclination vectors is always characterized by rank equal to 4 , and a high accuracy can be achieved.

Table 2: Observability properties for the relative orbits of Table 1, for the Keplerian problem.

\begin{tabular}{ccccc}
$\mathrm{RO}$ & $\delta a$ & $\delta u$ & $\operatorname{rank} \mathbf{H}$ & $\operatorname{cond}\left(\mathbf{H}^{\mathrm{T}} \mathbf{H}\right)$ \\
\hline 1 & $\mathrm{x}$ & $\mathrm{x}$ & 5 & $1.6^{19}$ \\
& $\mathrm{x}$ & - & 5 & 22.94 \\
& - & $\mathrm{x}$ & 4 & $1.9^{18}$ \\
& - & - & 4 & 1.06 \\
\hline 2 & $\mathrm{x}$ & $\mathrm{x}$ & 5 & $2.7^{18}$ \\
& $\mathrm{x}$ & - & 5 & 22.75 \\
& - & $\mathrm{x}$ & 5 & $1.2^{6}$ \\
& - & - & 4 & 1.06 \\
\hline 3 & $\mathrm{x}$ & $\mathrm{x}$ & 5 & $3.7^{18}$ \\
& $\mathrm{x}$ & - & 5 & 29.45 \\
& - & $\mathrm{x}$ & 4 & $1.4^{18}$ \\
& - & - & 4 & 1.33 \\
\hline 4 & $\mathrm{x}$ & $\mathrm{x}$ & 5 & $\infty$ \\
& $\mathrm{x}$ & - & 5 & 24.33 \\
& - & $\mathrm{x}$ & 4 & $\infty$
\end{tabular}

In our work the matrix of the dynamics of the system $\mathbf{A}$ is not exactly the transformed of the HCW equations: it includes also the first order effects due to the Earth equatorial bulge. As discussed before, these produce secular variations in four components of the mean relative orbital elements set, proportional to the $J_{2}$ value and the elapsed time $\Delta t$. By including these phenomena in the description of the dynamics, the observability properties of the problem can improve. To support this topic, in Table 3 are collected the results achieved by the same $\mathrm{RO}$ analyzed before, when the effects of $J_{2}$ are accounted for. Here two situations are compared: the difference consists in how $\mathbf{H}$ is generated. In the first column 6 measures spaced by $\Delta u=30 \mathrm{deg}$ are used, as accomplished for Table 2. In the second column the six measurements are separated by $60 \mathrm{deg}$, therefore spanning a double $\Delta t$. The aim is to evaluate the improvement of the observability when effects of $J_{2}$ becomes more relevant. One can note that RO1 and 2 are now characterized by rank 6 . Still the condition number is quite high, index of poor accuracy of the achievable estimation. As expected performances improve with the passing of the time. Regarding RO3 and 4, the $J_{2}$ cannot help as long as $\delta i_{x}$ is zero (the satellites lay on orbits of same inclination).

To conclude this session, the parameterization through relative orbital elements reveals convenient also when dealing with the analysis of the maneuvers needed to ensure observability. Performances are assessed through different metrics, that are discussed in the sequel. Table 4 resumes the effects of impulsive maneuvers on the set of relative orbital elements in accordance with Eq.(12). It is straightforward to verify that whatever 
Table 3: Observability properties for the relative orbits of Table 1, including $J_{2}$ effects.

\begin{tabular}{c|cc|cc} 
RO & \multicolumn{2}{|c|}{$\Delta t_{1}$} & \multicolumn{2}{|c}{$\Delta t_{2}$} \\
& $\operatorname{rank}(\mathbf{H})$ & $\operatorname{cond}\left(\mathbf{H}^{\mathrm{T}} \mathbf{H}\right)$ & $\operatorname{rank}(\mathbf{H})$ & $\operatorname{cond}\left(\mathbf{H}^{\mathrm{T}} \mathbf{H}\right)$ \\
\hline 1 & 6 & $6.35^{15}$ & 6 & $3.41^{14}$ \\
2 & 6 & $3.78^{15}$ & 6 & $1.61^{14}$ \\
3 & 5 & $2.62^{18}$ & 5 & $1.76^{19}$ \\
4 & 5 & $\infty$ & 5 & $\infty$
\end{tabular}

maneuver is performed the geometry of the relative motion varies in such a way that the natural trend of the LOS is modified.

Table 4: Effect of maneuvers on the relative orbital elements and their derivatives and, consequently on the angles to the LOS, given the attitude of Eq.(19). Variations in brackets stand for indirect effects via $\delta r$ and $\delta u$.

\begin{tabular}{cccc}
$d \mathbf{v}$ direction & \multicolumn{2}{c}{ Instantaneous effect on } & Effect on measures \\
& $\delta \boldsymbol{\alpha}$ & $\delta \dot{\boldsymbol{\alpha}}$ & \\
\hline$d v_{\mathrm{R}}$ & $\delta \mathbf{e}, \delta u$ & & $\Delta \eta,(\Delta \psi)$ \\
$d v_{\mathrm{T}}$ & $\delta a, \delta \mathbf{e}$ & $\delta \dot{u}$ & $\Delta \eta,(\Delta \psi)$ \\
$d v_{\mathrm{N}}$ & $\delta \mathbf{i}, \delta u(\operatorname{not} \delta \lambda)$ & & $\Delta \psi,(\Delta \eta)$
\end{tabular}

\section{B. Maneuver planning to optimize observability}

Once assessed that the presence of at least a maneuver is enough to achieve the full observability of the relative orbit estimation problem, the next step consists in quantifying the level of observability that different maneuver profiles can provide. This topic is interesting as long as the degree of observability is related to the accuracy achievable in the estimation. Moreover, during a rendezvous it can be useful to perform more effective maneuvers at the beginning, when errors in the estimations are bigger and when the large separation determines a less favorable situation. In Ref. 8 the authors suggest that the greater the LOS profile change produced by the maneuver, the higher the level of observability of the system, as the amount of change in the angle measurements is related to the range value before the maneuver. They extend further this topic in Ref. 9 defining a metric of detectability range error to quantify the achievable range accuracy, hence the level of observability of the problem. The optimal maneuver profile is the one that minimizes this metric. The range error, once fixed the accuracy of the sensor, is shown to be proportional to the change in position due to the maneuver and to the reciprocal of the sinus of the angle between the natural and modified LOS profiles. Therefore, assuming for example a servicer satellite in a positive V-bar station keeping, i.e. $a \delta \boldsymbol{\alpha}=\left(0,0,0,0,0, a \delta u_{0}\right)$ according to our formulation, the optimal maneuver is to burn in positive radial direction (thus acting on $\delta \mathbf{e}$ and $\delta u$ ). Geometrically the result means to establish a relative motion that is characterized by maximum radial displacement and minimum tangential separation, assumed a fixed delta-v magnitude. Tangential maneuvers were not taken into account due to the a-priori practical constraint of not changing the energy of the servicer orbit (in the paper the initial separation is of $100 \mathrm{~m}$ which corresponds to the very final part of a rendezvous).

When mentioning the change in position due to a maneuver, it is meant the variation in the whole relative motion geometry, thus in the LOS natural trend, appreciable over orbit-period time spans which leads to a non-instantaneous observability. In this context the exploitation of relative orbital elements is of great benefit as it allows having an immediate idea of what changes will take place in the LOS behavior after a extended-time maneuver (see Table 4 and Eq.(12)). The same effect can be mapped directly on the measurements, by expressing azimuth and elevation as functions of the relative orbital elements. Given 
Eq.(1) and sensor attitude of Eq.(19), the measures are expressed as follows:

$$
\begin{aligned}
& \eta=\arctan \left(\frac{\delta a-\delta e_{x} \cos u-\delta e_{y} \sin u}{-2 \delta e_{x} \sin u+2 \delta e_{y} \cos u-\delta u-\delta i_{y} \cot i}\right) \\
& \psi=\arcsin \left(\frac{a \delta i_{x} \sin u-a \delta i_{y} \cos u}{\|\delta \mathbf{r}\|}\right)
\end{aligned}
$$

In absence of maneuvers, these angles are orbital-period periodic functions whose amplitudes are related to the geometry of the motion. Whenever a maneuver takes place at $u_{M}$, the relative motion changes shape affecting the behavior of Eq.(23). This suggests a simple metric of observability based on the maximum amount of change in the measurement angles, according to:

$$
m_{1}=\left(\frac{1}{\kappa}\right) \cdot \max _{u \in\left[u_{M}, u_{M}+2 \pi\right]}\left|\xi_{\text {forced }}(u)-\xi_{\text {natural }}(u)\right|
$$

Exploiting the decoupling of the problem, $\xi$ is azimuth for in-plane maneuvers and elevation for out-of-plane maneuvers; $\kappa$ is the one-pixel angular resolution of the sensor expressed in radians. The metric $m_{1}$ allows assessing the minimum delta-v magnitude needed to obtain observability. It is the one that allows $m_{1}$ to overcome the instrument minimum sensitivity scale.

Metric $m_{1}$ can be rewritten as a function of the relative orbit before the maneuver. Let the ratio $N / D$ represent the argument of the inverse trigonometric functions of Eq.(23) then, to the $1^{\text {st }}$ order approximation, the variation of the measurements angles is proportional to the variation of the quantity $\Delta(N / D)$. This last term can be written as:

$$
\Delta(N / D)=\frac{N}{D} \cdot\left(\frac{\frac{\Delta N}{N}-\frac{\Delta D}{D}}{1+\frac{\Delta D}{D}}\right)
$$

therefore the maximum of the variation of the measurements angles is given by:

$$
m_{2}=\max _{u \in\left[u_{M}, u_{M}+2 \pi\right]}|\Delta(N / D)|
$$

Here pre-maneuver quantities, i.e. $N$ and $D$, are functions of $u$, whereas $\Delta \bullet$ quantities are constant finite variations determined by the type and size of the extended-time maneuver. In particular, $N$ stands for $\delta r_{x}^{\text {c }}$ or $\delta r_{y}^{c}$; $D$ for $\delta r_{z}^{\mathrm{c}}$ or $|\delta r|$, respectively for in-plane and out-of-plane cases. This metric is not defined when the pre-maneuver relative orbit either has $\delta a=|\delta \mathbf{e}|=0$ or $|\delta \mathbf{i}|=0$, respectively before in-plane and out-of-plane maneuvers.

Finally, Eq.(23) can be used to express how the accuracy of the measurements, i.e. $\sigma_{\eta}$ and $\sigma_{\psi}$, influence the achievable accuracy in $\delta u$ and $\delta r$ when a given relative motion is established.

$$
\begin{aligned}
\sigma_{\delta u}^{\text {post-man }} & =\frac{ \pm \sigma_{\eta}\left(\tan \eta \cdot \delta r_{x}^{\mathrm{c}}+\delta r_{z}^{\mathrm{c}}\right)}{\tan \eta \pm \sigma_{\eta}} \\
\sigma_{\delta r}^{\text {post-man }} & =\frac{\mp \sigma_{\psi} \cdot\|\delta \mathbf{r}\| \cdot \cos \psi}{\sin \psi \pm \sigma_{\psi} \cos \psi}
\end{aligned}
$$

In the development of these expressions it is assumed that the final orbital elements are perfectly known and the only source of error is the uncertainty in the angular measurements. These measurements errors are small angles and only $1^{\text {st }}$ order contributions are kept. As expected the achievable accuracies are function of the separation between the satellites. Similarly to Ref. 9 the problem of choosing the optimal maneuvers to improve observability can be formulated as the selection of that maneuver that brings to the minimization of the final achievable separation accuracy:

$$
m_{3}=\left\{\begin{array}{l}
\min _{u \in\left[u_{M}, u_{M}+2 \pi\right]}\left|\sigma_{\delta u}^{\text {post-man }}\right| \\
\min _{u \in\left[u_{M}, u_{M}+2 \pi\right]}\left|\sigma_{\delta r}^{\text {post-man }}\right|
\end{array}\right.
$$

Let us consider again the RO collected in Table 1, where RO4 is the example discussed in Ref. 9. Table 5 collects the scores achieved by the three metrics when maneuvers with fixed delta-v size are performed starting 
from RO1-4 initial relative orbits. In addition to the type and magnitude of the maneuver, all the metrics are also function of the verse and location of the maneuver. The values here presented simply refer to $u_{\mathrm{M}}=0$ deg (i.e., ascending node of servicer orbit). The verses are chosen to provide a reduction of the separation between the satellites. The magnitude of the maneuvers is $0.01 \mathrm{~m} / \mathrm{s}$. The instrument resolution is set equal to 0.024 deg per pixel. It is emphasized that these computations do not take into account constraints on the field of view of the instrument and on safety.

As expected, performances improve with the decreasing of the separation (from RO1 to RO4). At big separations, tangential maneuvers are more effective in terms of detectability of the maneuver by the instrument $\left(m_{1}\right.$ and $\left.m_{2}\right)$. Regarding the optimal value of the achievable accuracy $\left(m_{3}\right)$, one should investigate also the location of the maneuver. Concerning RO4, $m_{2}$ is not applicable as mentioned before. The value of $m_{1}$ could be rescaled as long as the instrument resolution used might not be appropriate for such a small relative separation. The tangential maneuver is shaded due to safety constraints: it will lead to collision in one orbit. In configuration RO4 metrics do not depend on $u_{M}$ and $\delta r=a \delta u$. Thus, in agreement with Ref. 9 a positive radial maneuver is the optimal one with respect to the achievable range accuracy. Finally, it is emphasized that the inclusion of safety and visibility constraints in the optimization problems is straightforward when using relative orbital elements. In Ref. 14 one can derive their explicit expressions.

Table 5: Values assumed by the metrics when maneuvers of $0.01 \mathrm{~m} / \mathrm{s}$ of magnitude are performed at $u_{M}=0$ deg.

\begin{tabular}{c|ccc|ccc|ccc} 
RO & \multicolumn{2}{|c|}{$\Delta v_{\mathrm{R}}=\Delta v \cdot(1 ; 0 ; 0)$} & \multicolumn{3}{c|}{$\Delta v_{\mathrm{T}}=\Delta v \cdot(0 ; 1 ; 0)$} & \multicolumn{3}{c}{$\Delta v_{\mathrm{N}}=\Delta v \cdot(0 ; 0 ; 1)$} \\
& $m_{1}$ & $m_{2}$ & $\sigma_{\delta u}$ & $m_{1}$ & $m_{2}$ & $\sigma_{\delta u}$ & $m_{1}$ & $m_{2}$ & $\sigma_{\delta r}$ \\
& {$[\mathrm{pixels}]$} & {$[\mathrm{ad}]$} & {$[\mathrm{m}]$} & {$[\mathrm{pixels}]$} & {$[\mathrm{ad}]$} & {$[\mathrm{m}]$} & {$[\mathrm{pixels}]$} & {$[\mathrm{ad}]$} & {$[\mathrm{m}]$} \\
\hline 1 & 0.78 & $3.3 e-4$ & 186.80 & 2.96 & $1.2 e-3$ & 187.45 & 0.78 & $3.3 e-4$ & 175.48 \\
2 & 1.19 & $0.5 e-3$ & 83.77 & 4.47 & $1.9 e-3$ & 82.65 & 1.17 & $4.9 e-4$ & 102.41 \\
3 & 6.76 & $2.8 e-3$ & 3.87 & 28.92 & $1.1 e-2$ & 3.07 & 7.56 & $3.2 e-3$ & 2.91 \\
4 & 288.07 & N.A. & 0.05 & $>3 e 3$ & N.A. & 0.003 & 226.98 & N.A. & 0.09
\end{tabular}

\section{Observability of the complete estimation problem}

According to the definition of observability, the pair of matrixes $(\hat{\mathbf{A}}(t), \hat{\mathbf{C}}(t))$ of the linear time variant (LTV) system of equation Eq.(2), is observable on the time interval $\left[t_{0}, t_{n}\right]$, if whatever the initial state and the system input, one can uniquely determine $\mathbf{x}_{0}$ from $\mathbf{y}(t)$ and $\Delta \mathbf{v}(t)$. Moreover, the zero state response contribution does not play any role in the observability characteristics, as it is a known term directly computed from $\Delta \mathbf{v}(t)$ and $\hat{\mathbf{\Phi}}$. The observability gramian for the pair $(\hat{\mathbf{A}}(t), \hat{\mathbf{C}}(t))$ is defined as:

$$
\mathbf{W}_{o}\left(t_{0}, t_{n}\right)=\int_{t_{0}}^{t_{n}} \hat{\boldsymbol{\Phi}}\left(\tau, t_{0}\right)^{\mathrm{T}} \hat{\mathbf{C}}(\tau)^{\mathrm{T}} \hat{\mathbf{C}}(\tau) \hat{\boldsymbol{\Phi}}\left(\tau, t_{0}\right) d \tau
$$

And the observability of the LTV system is equivalently stated by $\operatorname{det} \mathbf{W}_{o}\left(t_{0}, t_{n}\right) \neq 0$.

By passing in the discrete time domain, the observability gramian can be written as:

$$
\mathbf{W}_{o}\left(t_{0}, t_{n}\right)=\sum_{k=0}^{n}\left[\hat{\mathbf{\Phi}}\left(t_{k}, t_{0}\right)^{\mathrm{T}} \hat{\mathbf{C}}_{k}^{\mathrm{T}}\right] \cdot\left[\hat{\mathbf{C}}_{k} \hat{\mathbf{\Phi}}\left(t_{k}, t_{0}\right)\right]=\mathbf{H}^{\mathrm{T}} \mathbf{H}
$$

This corresponds to the normal matrix of the least squares solution of the initial-state determination problem when measurements are affected by observation errors. ${ }^{21}$ Here the matrix $\mathbf{H}$ represents the accumulated partials of the measures w.r.t. the initial state for the system with the variable state that includes the biases. To achieve observability, the gramian matrix has to be positive definite, i.e. $\mathbf{H}$ shall be full rank. Given Eq.(7) and Eq.(11), each sub-block associated to a i-th time has the following structure:

$$
\mathbf{H}_{i}=\left(\begin{array}{cc}
\mathbf{C}_{i} \boldsymbol{\Phi}\left(t_{i}, t_{0}\right) & \nu_{i} \mathbf{I}_{2 x 2}
\end{array}\right)
$$


which leads to this i-th addendum of the sum:

$$
\mathbf{W}_{o, i}\left(t_{0}, t_{i}\right)=\left(\begin{array}{cc}
\boldsymbol{\Phi}\left(t_{i}, t_{0}\right)^{\mathrm{T}} \mathbf{C}_{i}^{\mathrm{T}} \mathbf{C}_{i} \boldsymbol{\Phi}\left(t_{i}, t_{0}\right) & \nu_{i} \mathbf{C}_{i} \boldsymbol{\Phi}\left(t_{i}, t_{0}\right) \\
\nu_{i} \boldsymbol{\Phi}\left(t_{i}, t_{0}\right)^{\mathrm{T}} \mathbf{C}_{i}^{\mathrm{T}} & \nu_{i}^{2} \mathbf{I}_{2 x 2}
\end{array}\right)
$$

Thus the introduction of the biases in the estimation state does not vary the rank of $\mathbf{H}$. Moreover the eigenvalues of the gramian matrix associated to the biases are for sure positive $(\nu>0)$. In other words, the observability issues are confined in the reconstruction of the relative state from the available anglesmeasurements.

Whenever the observations errors have zero-mean time-uncorrelated characteristics, the observability properties are expressed by the following information matrix $\boldsymbol{\Lambda}:{ }^{21}$

$$
\boldsymbol{\Lambda}=\mathbf{H}^{\mathrm{T}} \widetilde{\mathbf{W}}^{-1} \mathbf{H}
$$

where the weighting matrix $\widetilde{\mathbf{W}}=\operatorname{diag}\left(\mathbf{W}_{1}, \cdots, \mathbf{W}_{n}\right)$ is the accumulated error covariance matrix and accounts for different accuracies of the angle measurements within the same data batch. In this situation the quantity $\mathbf{P}=\boldsymbol{\Lambda}^{-1}$ represents the covariance matrix of the estimation error associated to the least squares estimated state. Therefore $\mathbf{P}$ is related to the accuracy of the obtained estimate: the larger the elements of the matrix, the less accurate the correspondent components of the estimated state. ${ }^{21}$ In Ref. 10 the covariance of the relative position and velocity estimation has been used to define a metric of observability of the problem.

The introduction of the biases of the sensors into the estimation state leads to an information matrix that is numerically badly conditioned. Thus the estimation of the relative dynamics together with the biases is a weakly observable problem. The availability of some a-priori information related to the reference state used in the linearization process can aid to handle this numerical issue. The total information matrix of the problem becomes:

$$
\boldsymbol{\Lambda}_{\text {tot }}=\mathbf{H}^{\mathrm{T}} \widetilde{\mathbf{W}}^{-1} \mathbf{H}+\mathbf{P}_{a p r}^{-1}
$$

A non singular a-priori information matrix is sufficient to ensure that $\operatorname{det} \boldsymbol{\Lambda}_{t o t} \neq 0 .{ }^{22}$ In particular, by giving a small a-priori weight to the relative state (that is assuming a big standard deviation of the a-priori state) a small quantity will be added to the diagonal of $\boldsymbol{\Lambda}$. Therefore $\boldsymbol{\Lambda}_{\text {tot }}$ becomes a quasi-diagonal matrix, with great improvement of its conditioning characteristics. Moreover the level of confidence related to the a-priori state can be exploited to weight differently the effort in estimating different components. This aspect is particularly fruitful when an independent calibration system is able to provide a good estimation of the biases of the sensors. In that case, the accurate knowledge translates in small biases-standard deviations, thus the estimation problem focuses more on the relative state; fully observable in the presence of maneuvers. On the other side, if a true knowledge of the relative state is available, some a-posteriori characterization of the sensors can be accomplished by weighting more the, almost perfectly known, $\delta \boldsymbol{\alpha}$ components.

\section{Filter Design}

The estimation problem defined by the linear relative dynamics of solution Eq.(3) and nonlinear measurement equations of Eq.(1) is solved via a least-squares (LSQ) methodology. In the context of a ground-base scenario, this choice is motivated by the possibility to process at the same time the complete history of data thus allowing an efficient data editing. Moreover a deeper understanding of the systematic errors could be achieved as modeling errors are not absorbed by the process noise.

The least-squares relative orbit determination aims at finding the state $\mathbf{x}_{0}^{l s q}$ that minimizes the weighted squared sum of the difference between the actual measurements $\boldsymbol{\zeta}=\left(\boldsymbol{\zeta}_{0}, \cdots \boldsymbol{\zeta}_{t}\right)^{\mathrm{T}}$ and the modeled observations $\mathbf{z}$ of Eq.(1). ${ }^{21}$ Thus the loss function to be minimized is the following function of the observation residual $\rho$ :

$$
\begin{aligned}
J\left(\mathbf{x}_{0}\right) & =\boldsymbol{\rho}^{\mathrm{T}} \boldsymbol{\rho} \\
\boldsymbol{\rho}^{\mathrm{T}} & =(\boldsymbol{\zeta}-\mathbf{z})^{\mathrm{T}}=\left(\boldsymbol{\zeta}_{1}^{\mathrm{T}}-\mathbf{z}_{1}^{\mathrm{T}}, \cdots, \boldsymbol{\zeta}_{n}^{\mathrm{T}}-\mathbf{z}_{n}^{\mathrm{T}}\right)
\end{aligned}
$$

Here the $2 \mathrm{D}$ vector measurements of the batch are indexed through $i=(1, \cdots, n)$, where $n$ represents the number of processed images taken at times $t_{1}, \cdots, t_{n}$.

Due to the nonlinearity of the measurement equations, it is not possible to locate the absolute minimum of Eq.(35). A local minimum however can be found in the proximity of a reference state $\mathbf{x}_{0}^{r e f}=\mathbf{x}_{0}^{a p r}$, 
normally available within the application context. In addition to that, information on the accuracy of this reference value is usually also available, in the form of the a-priori covariance $\mathbf{P}^{\text {apr }}$ of the state. Such further piece of information can be incorporated into the least-squares estimation which, finally, aims at minimizing the following performance index:

$$
J\left(\mathbf{x}_{0}\right)=\boldsymbol{\rho}^{\mathrm{T}} \boldsymbol{\rho}+\left(\mathbf{x}_{0}-\mathbf{x}_{0}^{a p r}\right)^{\mathrm{T}} \boldsymbol{\Lambda}^{a p r}\left(\mathbf{x}_{0}-\mathbf{x}_{0}^{a p r}\right)
$$

As a result, the estimation is accomplished through an iterative dynamics batch least-squares estimator with a-priori information. ${ }^{22}$ The estimation state which best fits the observations in a least-squares of the residuals sense, or in other words, which minimizes the loss function defined by Eq.(36), is given by the iterations

$$
\begin{aligned}
\mathbf{x}_{j+1}^{l s q} & =\mathbf{x}_{j}^{l s q}+\left(\boldsymbol{\Lambda}^{a p r}+\mathbf{H}_{j}^{\mathrm{T}} \widetilde{\mathbf{W}}^{-1} \mathbf{H}_{j}\right)^{-1}\left[\boldsymbol{\Lambda}^{a p r}\left(\mathbf{x}_{j}^{l s q}-\mathbf{x}_{0}^{a p r}\right)+\mathbf{H}_{j}^{\mathrm{T}} \widetilde{\mathbf{W}^{-1}} \boldsymbol{\rho}_{j}\right] \\
\mathbf{P}_{j+1}^{l s q} & =\left(\boldsymbol{\Lambda}^{a p r}+\mathbf{H}_{j}^{\mathrm{T}} \widetilde{\mathbf{W}}{ }^{-1} \mathbf{H}_{j}\right)^{-1}
\end{aligned}
$$

which are started from $\mathbf{x}_{0}^{l s q}=\mathbf{x}_{0}^{a p r}$ and continued until convergence, typically after 4-5 iterations.

It is emphasized that the estimation time is the time at which the filter is initialized. Thus it could correspond either to the earliest time of the batch $\left(t_{1}\right)$ or to the latest one $\left(t_{n}\right)$. As a consequence the propagation of the state has to be accomplished coherently with the direction of changing of the time. This is achieved by design in Eqs.(10) and (11).

\section{Validation via Simulations}

In order to validate this relative navigation tool a modeling and simulation environment has been developed making use of the libraries and functionalities of the Multi-Satellite-Simulator developed at DLR/GSOC to support various projects in the field of multi-satellites applications. ${ }^{12}$ The purpose of this module is to emulate the behavior of the space segment of a two-satellites mission. Meanwhile the estimated delta-v of the performed maneuvers and the absolute state and attitude of the servicer satellite are logged. According to our filter design, these information are used to propagate the estimated relative state and to model the measurement observations $\mathbf{z}$ of Eq.(1).

The inertial orbits of both satellites are numerically integrated subjected to the gravity potential of a non homogeneous mass distribution of the $30^{\text {th }}$ order and degree, together with the main orbit perturbations acting in low energy orbits. The attitude mode of the servicer satellite is emulated. Maneuvers are introduced as time-tagged delta- $v$ which are afterwards translated into extended-time thrust burns. Regarding the simulations here discussed, the space systems are customized on the PRISMA mission. Therefore the satellites possess all the main characteristics of the Mango and Tango satellites, that respectively play the roles of servicer and client spacecraft. The model of the camera instrument also emulates the behavior of the Visual Based System (VBS) camera mounted on Mango, in terms of resolution, field of view, blinding angles, measurement noise and biases. Its output are the $x-y$ coordinates on the image plane according to Eq.(1). That coincides with the output that would be provided by an image processing unit elaborating real images. It is emphasized that the characteristics of the camera lens are not taken into account. The maneuvers profile provided to the simulation module is produced by a maneuver planning tool, which computes the ideal impulsive maneuvers that are needed to achieve a given relative formation, starting from the latest estimated relative state.

The main goal of the validation process is to assess the closed-loop-control accuracies achievable when performing a rendezvous ( $\mathrm{RdV}$ ) from far-range distance to a non-cooperative client satellite. To accomplish this the following realistic operational conditions are considered:

- The whole simulation is settled on the true orbit passes plan generated from the Tango orbit information available at the time. In particular the far-range RdV here discussed was scheduled between the 23Apr-2012 and the 27-Apr-2012. Eventual Moon and Sun eclipses are accounted for.

- Constraints related to typical operational day are included. They involve the location of the delta-v opportunities and the presence of data gaps in the measurements. These gaps can be caused either by mass memory and TM downlink limitations or by eclipses and instrument temporary malfunctioning. Regarding the RdV here presented, a data gap of about 7 hours, ending approximately around 14:00 
UTC, is introduced daily. It could represent the effect of a repositioning of the mass memory reading pointer so that the latest available TM refers to the current state of the space segment.

- The camera model provides measurements every $30 \mathrm{~s}$. Such sampling interval represents typical time frequencies of the image processing unit output, when exploiting relative target and stars movements from picture to picture.

- Measurements biases are included.

- When computing the extended-time thrust profile correspondent to the ideal delta-v provided by the maneuver planning tool, errors in the execution of the maneuver are introduced. Moreover the estimated delta-v that are logged during the simulation include also the errors accomplished by the estimation process. Aa a result the information used within the filter to model the measurement observations is affected by both maneuver execution and estimation uncertainties.

In addition to these operational constraints, in order to emulate an approach to a non-cooperative client satellite, the whole simulation is structured as follows. Each main task, i.e. maneuver planning, simulation and relative orbit determination, is accomplished by independent teams. In the sequel the relative orbit determination task will be referred as ROD. The simulation-specialist plays the role of the administrator of the $\mathrm{RdV}$ rehearsal. He is the only person aware of the true state of the client satellite, of the actual instrument biases and of the magnitude of maneuver execution and estimation errors. The simulation-specialist provides to the other teams an initial relative state and sets the aimed final hold-point of the rendezvous, e.g. Table 6. The error between true and provided initial conditions (IC) is of order of magnitude of the error committed when using NORAD TLE to compute the first-guess relative state. In the current simulation, biases were set of magnitude of about $1 / 8$ of pixel ( $\approx 10 \operatorname{arcsecs}$ ).

The rehearsal of the whole RdV develops through several steps, i.e. iterations, each composed by an execution of maneuver planning, simulation and relative orbit determination tasks. The objective is to refine step-wise the estimation of the relative state between the satellites. The process starts from the provided IC. Afterwards, during each iteration, given the latest estimation of the relative state, the maneuver planning module provides the next maneuvers to be accomplished. These are commanded to the simulation environment, that applies their effect on the space segment. The new acquired TM data together with the maneuver profile are subsequently used to perform a ROD run on the new data batch to update the estimation of the relative state, thus completing an iteration. As a result the errors committed by the relative orbit determination influence the performances of the maneuver plan. Errors in execution of the maneuvers affect the result of the aimed maneuvers. Therefore the complete functional chain allows assessing the realistic closed-loop-control performances of the rendezvous.

Table 6: Initial conditions (IC) provided to the ROD-team. They refer to MOS initial time: 23-Apr-2012 $14: 30: 14)$.

\begin{tabular}{lrrrrrr} 
Elements' set & \multicolumn{7}{c}{$a \delta \boldsymbol{\alpha}[\mathrm{m}]$} \\
\hline Provided IC & -1.00 & -41.16 & -377.60 & 19.53 & 246.50 & -30658.14 \\
(Unknown) true relative state & -9.32 & -79.24 & -395.29 & 46.42 & 557.99 & -30179.70 \\
(Unknown) error at initial time & 8.33 & 38.07 & 17.69 & -26.89 & -311.48 & -478.44 \\
\hline Aimed final hold-point & 0.0 & 0.0 & -200.0 & 0.0 & 200.0 & -3730.0
\end{tabular}

During the RdV, the maneuver planning module computes step by step the ideal delta-v needed to track a specific guidance profile that is designed to bring the servicer from the provided IC to the aimed final conditions. Such profile satisfies the eccentricity/inclination vector separation criteria suggested in Ref. 20. In addition to that, the guidance profile shall account for visibility constraints of the camera, safety constraints of the formation, delta-v budget limitations, delta-v execution opportunities and maneuvers selection to improve the observability of the relative navigation problem. All these topics and the results discussed in section B lead to the choice to perform couples of along-track maneuvers and cross-track corrections during the most far apart phase of the approach. Combinations of radial-along-track maneuvers and cross-track burns to refine the out-of-plane motion are performed in the final part of the approach. Regarding the distribution of delta-v opportunities, it is here assumed that two windows are daily available. Therefore, at 
least two ROD runs per day can profit of the presence of new maneuvers. Figure 2 reports all the delta-v recorded in the TM for the RdV defined in Table 6. The values nearer to zero are due to the errors in the execution and estimation of the maneuvers. The total delta-v spent is of $\approx 0.87 \mathrm{~m} / \mathrm{s}$.

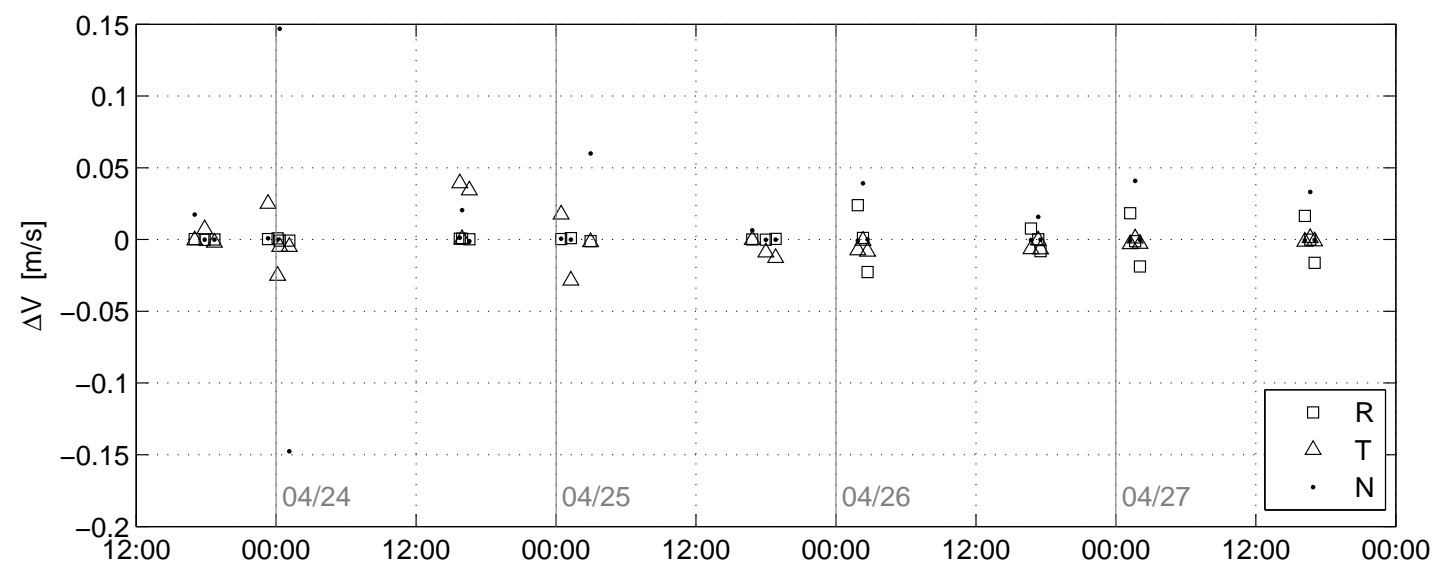

Figure 2: Estimated values of the maneuvers performed during the RdV of Table 6.

\section{A. Rendezvous simulation results}

In Table 7 are described all the ROD runs performed during the RdV from the initial conditions to the aimed final hold-point reported in Table 6. Runs are tagged with labels from R1 to R9. To each of them it is associated the latest active orbit contact, the time span covered by the measurements and the number of camera outputs used for the relative orbit determination. Due to how the measurements are modeled in the simulation environment, there is no need to perform any data editing. Therefore all the measures outside data gaps can be used by the filter. Runs R1-6 exploit all the available data till the latest time; whereas R7-9 use just the most recent 24 hours of data. The choice of which data batch to use comes from some analysis accomplished during the ROD module development. Moreover, the strategy was finally refined by tailoring it on the results gained by different simulation scenarios, though sharing the same guidance approach during the rendezvous.

Table 7: Summary of the ROD runs performed during the RdV.

\begin{tabular}{ccccc} 
ROD & $\begin{array}{c}\text { Pass \# } \\
{[\mathrm{ad}]}\end{array}$ & from [GPS time] & to [GPS time] & $\begin{array}{c}\text { D measures } \\
\text { [ad] }\end{array}$ \\
\hline R1 & 9767 & 23-Apr-2012 14:30:14 & 23-Apr-2012 19:28:44 & 598 \\
R2 & 9778 & 23-Apr-2012 14:30:14 & 24-Apr-2012 13:49:15 & 1991 \\
R3 & 9782 & 23-Apr-2012 14:30:14 & 24-Apr-2012 20:26:45 & 2786 \\
R4 & 9793 & 23-Apr-2012 14:30:14 & 25-Apr-2012 14:50:45 & 4185 \\
R5 & 9797 & 23-Apr-2012 14:30:14 & 25-Apr-2012 21:24:45 & 4973 \\
R6 & 9807 & 23-Apr-2012 14:30:14 & 26-Apr-2012 14:09:15 & 6175 \\
R7 & 9811 & 25-Apr-2012 19:47:54 & 26-Apr-2012 20:45:24 & 2189 \\
R8 & 9821 & 26-Apr-2012 14:09:23 & 27-Apr-2012 13:26:54 & 1956 \\
R9 & 9826 & 26-Apr-2012 19:08:43 & 27-Apr-2012 21:39:54 & 2321
\end{tabular}

As explained before in the filter design section, the LSQ filter can be initialized either at the initial or at the final time of the data batch (marked by a star in Figure 3). Depending on the verse of the time propagation, the filter can be labeled as forward (FW) or backward (BW). As the dynamics is modeled by a linear system and the LSQ approach fits the whole data set at once, the accuracy of the estimated state is 
the same, disregarding the verse of time propagation. In the case of sequential relative orbit determinations during the rendezvous, the filter is set to $B W$, as its output directly feeds the maneuver planning tool (structure depicted in the bottom part of the sketch in Figure 3).

Concerning how successive RODs are linked one
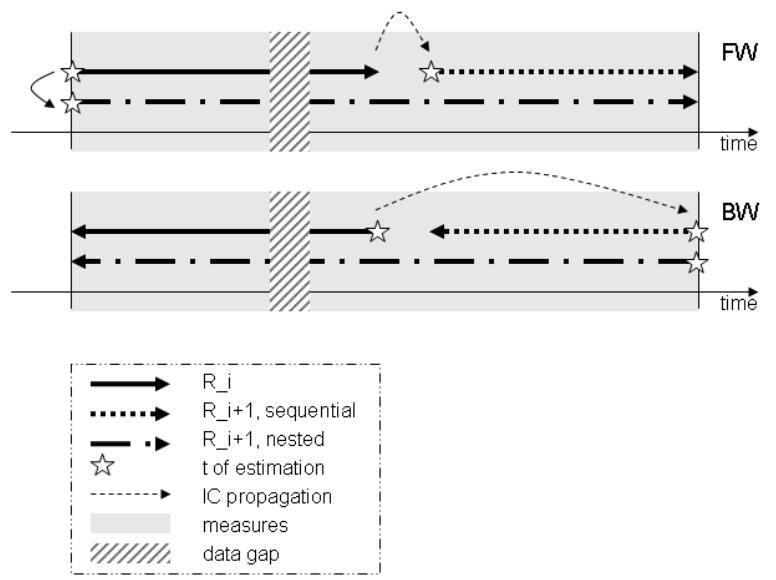

Figure 3: Schematic view of the LSQ approach and of the concatenations of successive ROD runs.

can focus on what data batches they use. Let bold arrows of Figure 3 symbolize each ROD run, i.e. estimation based on a given data batch. A i-th ROD, i.e. solid arrow, can be followed by one that uses just new data, i.e. sequential link marked with dotted arrow, or by one that overlaps new and old data in a nested way, i.e. dash-dotted arrow. For $B W$ LSQ, the estimation time will be the same, the data fitted by the nested link, however, will be more and distributed on a wider time span. Numerical simulation of different guidance profiles showed that a nested link should be preferred for the following reasons. The trends of estimation errors over consecutive ROD runs are less irregular for the nested link case. This aspect is important for the closed-loop control of the rendezvous, as ROD output can be considered reliable since the first steps. Unexpected bigger errors influence the MAP profile and, consequently, the $\Delta V$ budget to achieve the final target hold-point. Furthermore, when a nested approach is used, each ROD run can contain a greater equal number of maneuvers than the sequential run terminating with same estimation time. This aspect improves the degree of observability of the estimation problem. Finally, taking into account the ground-in-the-loop architecture, it is convenient to exploit at maximum the amount of available data; coherently with the choice to employ a LSQ approach.

The last issue involved in the definition of time spans in Table 7 is related to how big the nested data batch can become. The rule employed in the RdV was determined by comparing the magnitude of the estimation errors after each run when either all available data or most recent 24 hours were used, during previous rendezvous rehearsals. Within the simulation characteristics and the modeling limitations of this application, it turned out to be convenient to switch to shorter data batches from the second part of day- 4 . Regarding the investigated guidance profiles, at that time the average along-track separation was less than $4 \mathrm{~km}$. The maximum time span covered by the data batch should be traded off considering when the benefit of having an extensive sample of measurements is countered by the deterioration of the capability to model the measurements due to un-modeled phenomena over time. To this topic contribute the accuracy of the model of the relative dynamics and the behavior of the true observations over time. Our model of the relative dynamics does not include the differential drag effects (see Eq.(10)). In the simulation frame, the reality is represented by the numerical integration of the absolute dynamics of the two satellites. True observations are modeled disregarding many aspects of the instrument functioning. Therefore camera lens characteristics, different lighting conditions, dimension of the client spot and behavior of the centroiding function are not taken into account. As a consequence the true-measurements are always of the same quality during the $\mathrm{RdV}$, thus favoring the choice of data batches of wide time spans.

The initial condition for the biases was set to 0 radians. Due to the bad conditioning of the information matrix of the complete LSQ problem it is set that the filter cannot substantially modify the values of the estimated biases. This is accomplished by attributing an a-priori standard deviation to the biases significantly smaller than the one of the other parameters. In particular the following values were used during the $\mathrm{RdV}$ :

$$
\sigma_{b, \eta}=1 e^{-5}[\mathrm{deg}], \quad \sigma_{b, \psi}=1 e^{-7}[\mathrm{deg}]
$$

The standard deviation of the measurements, i.e. $\sigma_{\eta}=\sigma_{\psi}$, was set to $1 / 2$ pixel circa $(\approx 0.012 \operatorname{deg})$, coherently with the instrument noise employed in the MOS simulation. The consequent weight matrix $\mathbf{W}$ was kept constant throughout the whole simulation.

The remaining filter parameter of each run is the a-priori covariance matrix $\mathbf{P}^{a p r}$ computed from the a-priori standard deviation set for the relative orbital elements $\sigma_{\delta \boldsymbol{\alpha}}$. Table 8 lists all the sets employed 
Table 8: A-priori standard deviation of the relative orbital elements at each run.

\begin{tabular}{lrrrrrr} 
ROD & \multicolumn{7}{c}{$\sigma_{\delta \boldsymbol{\alpha}}=\operatorname{diag}\left(\mathbf{P}^{a p r}\right)[\mathrm{m}]$} \\
\hline R1 & 20.00 & 100.00 & 100.00 & 100.00 & 100.00 & 10.00 \\
R2 & 1.34 & 1.88 & 1.95 & 1.88 & 2.10 & 50.00 \\
R3 & 0.25 & 0.32 & 0.59 & 0.32 & 0.82 & 50.00 \\
R4 & 0.28 & 0.34 & 0.59 & 0.34 & 0.77 & 50.00 \\
R5 & 0.36 & 0.35 & 0.65 & 0.34 & 0.79 & 50.00 \\
R6 & 0.01 & 0.01 & 0.01 & 100.00 & 100.00 & 0.01 \\
R7 & 47.80 & 48.87 & 48.84 & 229.25 & 232.14 & 50.00 \\
R8 & 0.64 & 0.78 & 2.46 & 0.77 & 2.58 & 50.00 \\
R9 & 0.32 & 0.37 & 2.87 & 0.35 & 3.00 & 50.00
\end{tabular}

during each run of the RdV. In particular, in R $1 \sigma_{\delta \boldsymbol{\alpha}}$ represents the typical confidence that the provided IC, computed via TLE, can guarantee. In addition to that it was artificially set $\sigma_{\delta u}$ smaller than the one of the other elements, since the filter should not modify too much the related element of the initial guess state. This is motivated by the fact that during the flight time of $\mathrm{R} 1$ no maneuvers were executed, which reduced the degree of observability of the problem. By fixing the average along-track separation, the filter can act only on the remaining parameters which define the shape of the projection of the relative motion on the radial-cross-track $(R-N)$ plane. Given Eq.(19), camera boresight direction and mean tangential separation at the beginning of the rendezvous, the $R$ - $N$ plane almost coincides with the plane of the image of the camera. Thus the fitting leads to the identification of the family of the relative motion, with an error in the scale factor related to the ambiguity on $a \delta a, a \delta u$ and $b_{\eta}$. Nevertheless, the less accurate components of the initial guess from TLE, i.e. $a \delta \mathbf{e}$ and $a \delta \mathbf{i}$, can be efficiently adjusted [c.f. the third line of Table 6 and the R1 abscissa of Figure 5]. Such an early correction, accomplished without need of maneuvers, brings great benefit to the successive MAP planning. $\sigma_{\delta \boldsymbol{\alpha}}$ settings in R2-5 R7-9 are related to the choice of how to link the estimation information from run to run. The LSQ filter provides the covariance matrix of the least-squares solution according to Eq.(37). This quantity is function of the a-priori confidence and of the number of observations used. Therefore the magnitude of the elements of the obtained $\mathbf{P}^{l s q}$, i.e. the formal standard deviation of the estimated state, are not representative of the estimation error committed, depicted in Figure 5. Moreover it has been observed that by simply letting $\mathbf{P}^{l s q}$ to become the a-priori confidence of the initial conditions of next run, the filter reaches in few runs a saturation point. In particular the estimated state is trusted more than the fresh information brought by new measurements, leading the filter to diverge.

In order to avoid this phenomenon it has been established a rule to link the $\mathbf{P}^{l s q}$ to the a-priori confidence of the successive ROD run. A minimum-reference confidence $\sigma_{r e f-\min }$ is set, coherently with the expected order of magnitude of the estimation error in the final phases. During the simulation such quantity was fixed to:

$$
\sigma_{\text {ref-min }}=(3,10,10,10,10,50)[\mathrm{m}]
$$

Then, whenever any element was satisfying $\sigma_{\delta \boldsymbol{\alpha}}<\sigma_{r e f-\min }$, the whole set was rescaled so that its most violating term would be equal to its correspondent in $\sigma_{r e f-m i n}$. Thus, values reported in Table 8 are computed via the above mentioned rule, starting from the solution of Eq.(37) (elevation defined w.r.t. the local cross-track direction). In R6 a different decision was employed: the idea was to exploit the execution of the first radial maneuvers to refine the estimation of the biases. A radial maneuver in fact inserts a variation in the $a \delta u$, thus adding information to resolve the ambiguity on the scale factor of the projection of the motion on the $R-N$ plane. The bias in azimuth, is perceived as a shift in the radial direction, thus it couples itself with the estimation of $a \delta a$. The bias in elevation has no justification w.r.t. the solution of the relative dynamics, given the attitude of Eq.(19). 

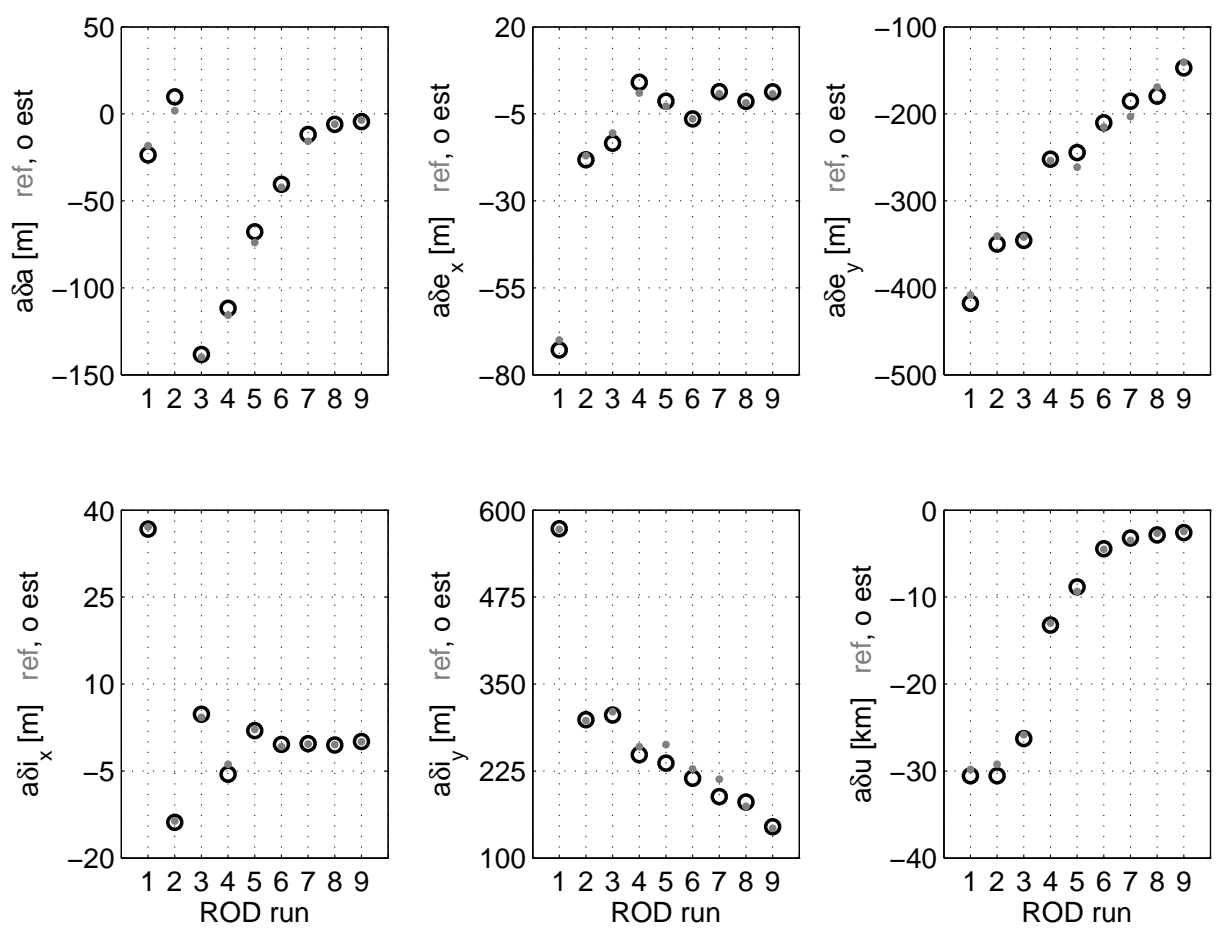

Figure 4: Estimated and reference relative orbital elements at the estimation time of each run.
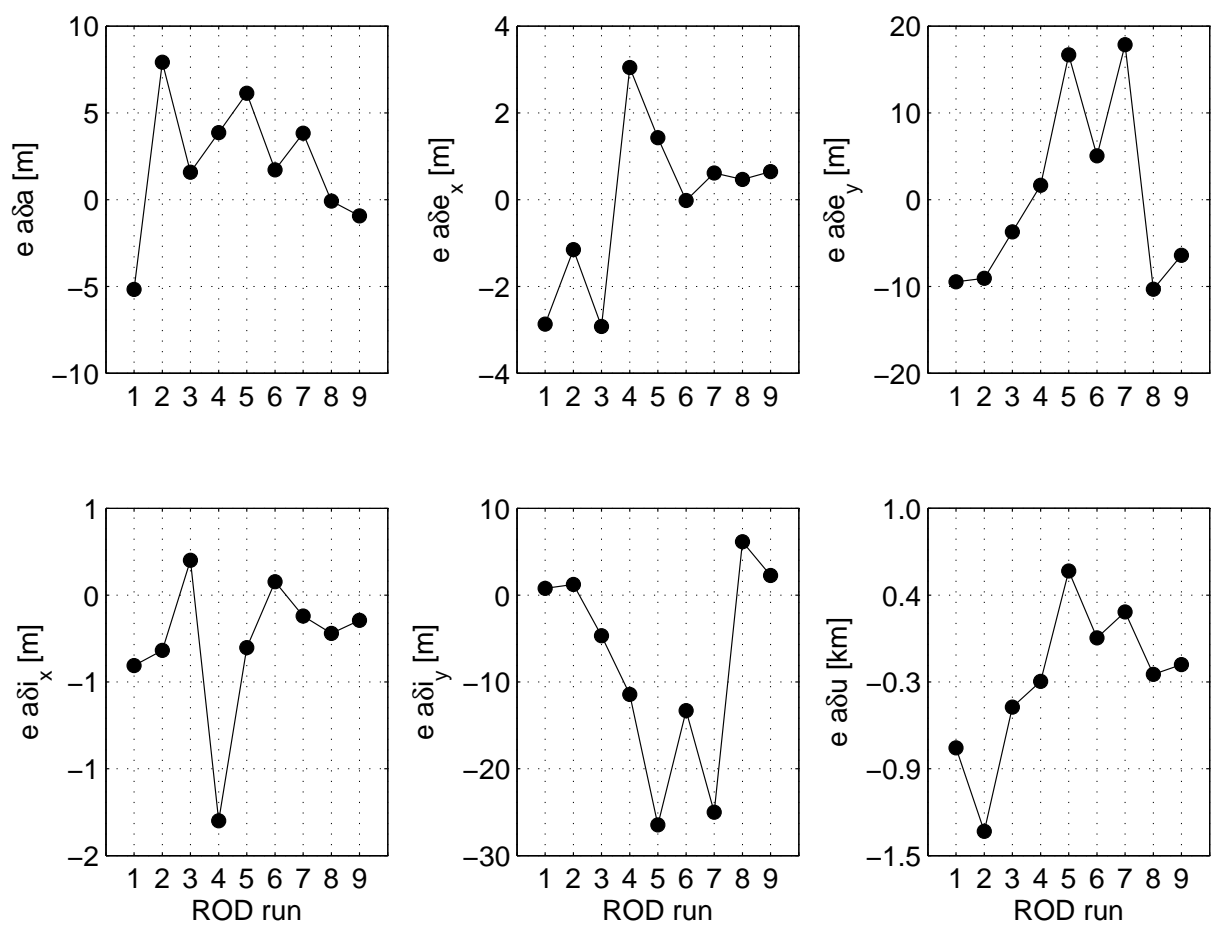

Figure 5: Estimation error at the estimation time of each run. 
To this aim a loop of runs were performed in which on turn only some elements were allowed to be adjusted. In particular the following sequence was executed:

1. $a \delta u$ was fixed (similarly to $\mathrm{R} 1$ ), in order to estimate shape and orientation of the relative motion;

2. all relative elements were fixed except $a \delta u$ and $b_{\eta}$, in order to refine the azimuth bias estimation;

3. $a \delta a$ and $\mathbf{b}$ were fixed, to refine shape and orientation w.r.t. updated values of the biases;

4. all fixed except $a \delta \mathbf{i}$; to refine the estimation of the relative inclination vector.

These last settings correspond to the ones reported in Table 8. For each bias, the true values set in the MOS simulator were of 10 arcsecs; in the current simulation the process just described did not improve the accuracy of the estimation of $\mathbf{b}$. It should be noted, however, that R6 achieved the minimum value of estimation error in $a \delta u$ (abscissa R6 in Figure 5). The effectiveness of this loop of runs varied across the different simulations performed. It revealed more efficient when both the biases in the MOS simulation and the error between biases initial-guess and true-value were bigger.

Figures 4 and 5, report the accuracies achieved component by component for each run of the RdV. The difference between the estimated state and the true one defines the estimation error committed at each step. These figures allow respectively understanding what was the rendezvous guidance strategy and with what accuracy it was achieved. Note that features regarding $a \delta u$ are plotted in $\mathrm{km}$. By observing Figure 5, one can note that the error committed in $a \delta a$ stays in $\pm 8 \mathrm{~m}$ during the rendezvous and decreases with the proceeding of the runs. Both $a \delta e_{x}$ and $a \delta i_{x}$ are well estimated. Their y-components are estimated with an error of $\pm 30 \mathrm{~m}$. The mean along-track separation is always estimated within an error minor than the $7.5 \%$ of the current true mean along-track separation, which spans from circa 30 to $3 \mathrm{~km}$.

Finally, Figure 6 and Table 9 report the characteristics of the observation residuals during the RdV. One can note that the residual in elevation, i.e. cross-track direction, is biased throughout the simulation. Regarding our experience, during all the simulations performed that shared the same sensor attitude, the bias in elevation has been estimated with less accuracy. Together with this, the residuals present a periodical pattern superposed to a not zero mean value that clearly is an evidence of some not modeled phenomenon that ultimately ends into the residuals. This is related to how the instrument is modeled in the simulation: information from absolute positions and attitude are used to reproduce the x-y coordinates of Tango on the image plane, therefore true measurements are not completely independent information. As a confirmation, if biases are set to 0 during the simulation, the behavior of the residuals obtained during a relative orbit determination phase, presents a mean trend around zero, and less marked oscillations.

\begin{tabular}{crr} 
ROD & \multicolumn{2}{c}{$\begin{array}{r}\text { Residuals mean } \pm \sigma \\
{[\operatorname{arcsecs}]}\end{array}$} \\
& \multicolumn{1}{c}{$\rho_{\eta}$} & \multicolumn{1}{c}{$\rho_{\psi}$} \\
\hline R1 & $0 \pm 66$ & $131 \pm 31$ \\
R2 & $4 \pm 53$ & $148 \pm 32$ \\
R3 & $-11 \pm 70$ & $140 \pm 37$ \\
R4 & $-1 \pm 70$ & $102 \pm 72$ \\
R5 & $1 \pm 68$ & $100 \pm 80$ \\
R6 & $1 \pm 59$ & $97 \pm 59$ \\
R7 & $14 \pm 84$ & $86 \pm 64$ \\
R8 & $2 \pm 56$ & $78 \pm 38$ \\
R9 & $-3 \pm 126$ & $101 \pm 96$
\end{tabular}

Table 9: Merit features of the residuals for each run of the RdV.
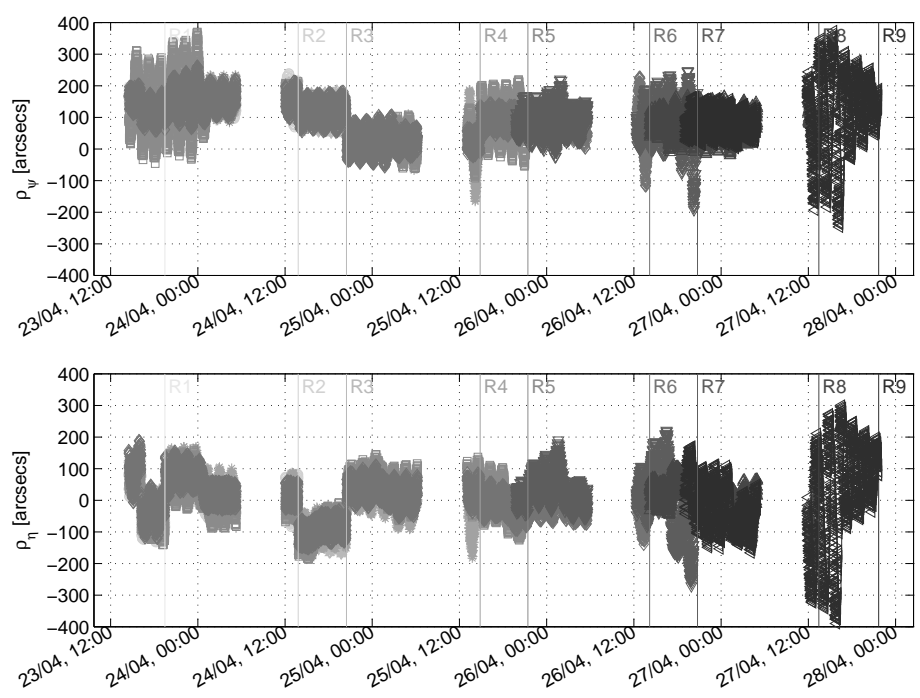

Figure 6: Behavior of the observation residuals over time, during each run of the RdV. 


\section{Conclusion}

This work addressed the design and the implementation of a relative navigation tool that makes use of camera-based angles-only measurements.

The first part of the paper dealt with the theoretical background of the estimation problem and with the subsequent filter design. The relative dynamics has been parameterized through relative orbital elements. This choice allowed exploiting a simple model of the dynamics which includes the effect of the Earth oblateness, that is one of the main perturbations in low energy orbits. Moreover the parameterization allowed having a direct physical interpretation of the range un-observability of the relative orbit determination using line-of-sight measurements. Observability properties were discussed for the Kepler and the perturbed problems. Given the need to perform a maneuver in order to achieve the full observability, in the paper has been introduced some possible metrics to quantify the subsequent level of observability. Such metrics exploit the simple relations between maneuvers and changes in the relative orbital elements. Finally it is discussed how the observability properties behave when sensor biases are included in the variable state.

The estimation problem of a far-range orbital rendezvous was solved with an iterative least-squares approach that makes use of a-priori information. The proposed relative orbit navigation tool has been validated within a high-fidelity simulation environment. There several realistic operational conditions have been included. Despite this, still some topics cannot be fully addressed through a simulation environment. The choice of the size of the data batches, for example, could be not optimal for a real-world application. This is related to the difference between the reality and the simplified simulation environment. Moreover the behavior of the true measurements over time, due to different illumination conditions and decreasing relative separation was not included in the simulation. Again this could lead to prefer shorter/longer data batches. Finally, in the simulation environment the behavior of the observation residuals revealed artificial and provided very little information compared to what it provides when real measurements coming from flight data are used.

The set up of a realistic rehearsal of a rendezvous from far-range distance to a non-cooperative client satellite allowed assessing the achievable closed-loop-control performances. It was demonstrated the feasibility of a rendezvous guidance profile based on (anti-)parallel eccentricity/inclination vectors whose magnitude shrinks with the passing of the time. Moreover it was assessed the robustness of the relative navigation tool with respect to realistic errors in execution and estimation of the maneuvers.

The development of this tool happened in the frame of the preparation to the DLR/GSOC Advanced Rendezvous Demonstration using GPS and Optical Navigation (ARGON) experiment. Future refinement will take advantage of the flight data collected during the execution of this experiment.

\section{References}

${ }^{1}$ D'Amico, S. and Spurmann, J., "Formation Flying Concept for DEOS," DLR-GSOC TN 10-07, Deutsches Zentrum für Luft- und Raumfahrt, Oberpfaffenhofen, July 2010.

${ }^{2}$ Persson, S., D'Amico, S., and Harr, J., "Flight Results from PRISMA Formation Flying and Rendezvous Demonstration Mission," 61 $61^{\text {st }}$ International Astronautical Congress, Prague, Czech Republic, 2010.

${ }^{3}$ D'Amico, S., Ardaens, J. S., and Larsson, R., "Spaceborne Autonomous Formation-Flying Experiment on the PRISMA Mission," Journal of Guidance, Control, and Dynamics, Vol. 35, No. 3, 2012, pp. 834-850.

${ }^{4}$ D'Amico, S., Autonomous Formation Flying in Low Earth Orbit, Ph.D. thesis, Technical University of Delft, The Netherlands, March 2010.

${ }^{5}$ D'Amico, S. and Montenbruck, O., "Proximity Operations of Formation Flying Spacecraft using an Eccentricity/Inclination Vector Separation," AIAA Journal of Guidance, Control and Dynamics, Vol. 29, No. 3, 2006, pp. 554-563.

${ }^{6}$ Nardone, S. C. and Aidala, V. J., "Observability Criteria for Bearings-Only Target Motion Analysis," IEEE Transactions on Aerospace and Electronic Systems, Vol. 17, No. 2, 1981, pp. 162-166.

${ }^{7}$ Hammel, S. E. and Aidala, V. J., "Observability Requirements for Three-Dimensional Tracking via Angle Measurements," IEEE Transactions on Aerospace and Electronic Systems, Vol. 21, No. 2, 1985, pp. 200-207.

${ }^{8}$ Woffinden, D. C. and Geller, D. K., "Observability Criteria for Angles-Only Navigation," IEEE Transactions on Aerospace and Electronic Systems, Vol. 45, No. 3, 2009, pp. 1194-1208.

${ }^{9}$ Woffinden, D. C. and Geller, D. K., "Optimal Orbital Rendezvous Maneuvering for Angles-Only Navigation," Journal of Guidance, Control, and Dynamics, Vol. 32, No. 4, 2009, pp. 1382-1387.

${ }^{10} \mathrm{Li}$, J. R., Li, H. Y., Tang, G. J., and et al., "Research on the strategy of angles-only relative navigation for autonomous rendezvous," Sci China Tech Sci, Vol. 54, 2011, pp. 1865-1872.

${ }^{11}$ Yim, J. R., Crassidis, J. L., and Junkins, J. L., "Autonomous orbit navigation of two spacecraft system using relative line of sight vector measurements," No. 04, 2004. 
${ }^{12}$ Gaias, G., Ardaens, J. S., and D'Amico, S., "Formation Flying Testbed at DLR's German Space Operations Center," 8th International ESA Conference on Guidance, Navigation \& Control Systems, Carlsbad, Czech Republic, 2011.

${ }^{13}$ Karlsson, T., Ahlgren, N., Faller, R., and Schlepp, B., "PRISMA Mission Control: Transferring Satellite Control between Organisations," SpaceOps 2012, Stockholm, Sweden, 2012.

${ }^{14}$ DAmico, S., Ardaens, J. S., Gaias, G., Benninghoff, H., Schlepp, B., and Jørgensen, J. L., "Non-Cooperative Rendezvous using Angles-only Optical Navigation: System Design and Flight Results," submitted to: Journal of Guidance, Control, and Dynamics.

${ }^{15}$ Clohessy, W. H. and Wiltshire, R. S., "Terminal Guidance System for Satellite Rendezvous," Journal of the Aerospace Sciences, Vol. 27, 1960, pp. 653-658.

${ }^{16}$ Brower, D., "Solution of the problem of artificial satellite theory without drag," Astronomical Journal, Vol. 64, No. 1274, 1959, pp. 378-397.

${ }^{17}$ Battin, R. H., An Introduction to the Mathematics and Methods of Astrodynamics Revised Edition, AIAA Education Series, 1999.

${ }^{18} \mathrm{Schmidt}$, J. and Lovell, T. A., "Estimating Geometric Aspects of Relative Satellite Motion Using Angles-Only Measurements," AIAA Paper, Vol. 6604, 2008.

${ }^{19}$ Sage, A. P., Optimum Systems Control, Prentice-Hall, 1968.

${ }^{20}$ Spurmann, J. and D'Amico, S., "Proximity Operations of On-Orbit Servicing Spacecraft using an Eccentricity/Inclination Vector Separation," 22nd International Symposium on Spaceflight Dynamics, Sao Jose dos Campos, Brazil, 2011.

${ }^{21}$ Tapley, B. D., Schutz, B. E., and Born, G. H., Statistical Orbit Determination, Elsevier Academic Press, 2004.

${ }^{22}$ Montenbruck, O. and Gill, E., Satellites Orbits - Models, Methods, and Applications, Springer Verlag, 2001. 